\title{
Legionella pneumophila induces cathepsin B-dependent necrotic cell death with releasing high mobility group box1 in macrophages
}

Yoshitomo Morinaga ${ }^{1,2}$, Katsunori Yanagihara ${ }^{1,2^{*}}$, Shigeki Nakamura², Hiroo Hasegawa', Masafumi Seki², Koichi Izumikawa², Hiroshi Kakeya², Yoshihiro Yamamoto ${ }^{2}$, Yasuaki Yamada', Shigeru Kohno ${ }^{2,3}$, Shimeru Kamihira ${ }^{1}$

\begin{abstract}
Background: Legionella pneumophila (LPN) can cause a lethal infectious disease with a marked inflammatory response in humans. However, the mechanism of this severe inflammation remains poorly understood. Since necrosis is known to induce inflammation, we investigated whether LPN induces necrosis in macrophages. We also analyzed the involvement of lysosomal cathepsin B in LPN-induced cell death.

Methods: The human monocytic cell line THP-1 was infected with LPN, NUL1 strain. MG132-treated cells were used as apoptotic control cells. After infection, the type of cell death was analyzed by using microscopy, LDH release and flow cytometry. As a proinflammatory mediator, high-mobility group box 1 (HMGB-1), was measured. Cathepsin B activity was also measured and the inhibitory effects of cathepsin B on LPN-induced cell death were analyzed.

Results: THP-1 cells after treatment with high dose of LPN showed necrotic features with releasing HMGB-1. This necrosis and the HMGB-1 release were inhibited by a specific lysosomal cathepsin B inhibitor and were characterized by a rapid and high activation of cathepsin B that was not observed in apoptotic control cells. The necrosis was also accompanied by cathepsin B-dependent poly(ADP-ribose) polymerase (PARP) cleavage.

Conclusions: We demonstrate here that L. pneumophila rapidly induces cathepsin B-dependent necrosis in a dosedependent manner and releases a proinflammatory mediator, HMGB-1, from macrophages. This report describes a novel aspect of the pathogenesis of Legionnaires' disease and provides a possible therapeutic target for the regulation of inflammation.
\end{abstract}

\section{Introduction}

Legionella pneumophila is an intracellular pathogen that causes rapidly advancing pneumonia and is sometimes life-threatening. After inhalation into the lung, the organism initially infects alveolar macrophages and replicates in these cells. The infected macrophages produce cytokines such as IL- $\beta$ and TNF- $\alpha$ that activate both themselves and other immune cells [1]. However, although the functions of macrophages in response to this pathogen are crucial for innate immunity, the

\footnotetext{
* Correspondence: kyana-ngs@umin.ac.jp

'Department of Laboratory Medicine, Nagasaki University Graduate School of Biomedical Sciences, 1-7-1 Sakamoto, Nagasaki, 851-2128, Japan
}

Full list of author information is available at the end of the article mechanism by which this pathogen induces such a severe immune response is not well understood.

In infectious diseases, cell death that occurs as a result of interactions between the infectious organism and the host cell can have important implications for host defense or bacterial survival. Apoptosis is a typical programmed cell death that is tightly regulated by various proteases, requires ATP and does not involve inflammation [2]. In contrast, necrosis, a type of cell death that is accompanied by inflammation, has been considered to represent accidental cell death due to exposure to supraphysiological conditions such as mechanical trauma, heat or cold [3]. During interactions between pathogens such as Shigella [4], Salmonella [5] and Mycobacterium tuberculosis [6] and the host immune response, there 
have been some reports of cell death induced by these bacteria that appears to have features of necrosis. While L. pneumophila has been shown to induce apoptosis in macrophages or monocytic cell lines when the cells were infected at a low dose of bacteria [7-9], induction of apoptosis is not necessarily associated with pathogenesis in severe infections. Thus, necrosis can contribute to inflammation in Legionnaires' disease, although there are few reports concerning the induction of necrosis by L. pneumophila, in which a high dose of bacteria was used $[10,11]$.

Recent research has implicated lysosomal function in cell death [12]. Many types of proteases and chemical agents that are known apoptosis inducers, such as caspases, anticancer agents and reactive oxygen species, may also be involved in cell death via the modulation of lysosomal membrane permeability, and some of these agents also induce necrosis [13]. Similarly, it has been shown that necrosis, like apoptosis, can be regulated by intracellular molecules, and lysosomes in particular are considered to be important organelles for programmed necrosis $[13,14]$.

In this report, we determined if L. pneumophila induces necrotic cell death in a monocytic cell line and in murine macrophages by comparing cell death induced by L. pneumophila with that induced by an apoptotic agent. We also examined the role of lysosomal enzymes in $L$. pneumophila-induced cell death. We found that potent activation of cathepsin B leads to necrosis accompanied by inflammation in cells infected with a high dose of L. pneumophila. In addition, cell death and inflammation were inhibited by attenuation of cathepsin B.

\section{Materials and methods Reagents}

PARP antibody was from Cell Signaling Technology (Danvers, MA) and anti-cathepsin B antibody (CA10) was from Abcam (Cambridge, MA). CA074Me and zVADfmk were obtained from the Peptide institute (Osaka, Japan).

\section{Bacterial strains}

The L. pneumophila NUL1 bacterial strain, serogroup 1, which was clinically isolated from the sputum of a patient at Nagasaki University Hospital [15], was used. The bacteria were cultured on buffered charcoal yeast extract $\alpha$ agar plates for 3 days. The bacteria were stored at $-80^{\circ} \mathrm{C}$ in a Microbank system (Pro-Lab Diagnostics, Ontario, Canada) until use.

\section{Animals}

Female A/J mice, 6-8-weeks old, were purchased from Japan SLC, Inc. (Shizuoka, Japan). All animals were housed in a pathogen-free environment and received sterile food and water in the Laboratory Animal Center for Biomedical Science at Nagasaki University. Animal care and experimental procedures were performed in accordance with the Guidelines for Animal Experimentation of Nagasaki University with approval of the Institutional Animal Care and Use Committee.

\section{Cell lines and treatment procedure}

The human monocytic cell line THP-1 was cultured in RPMI1640 medium containing 10\% FBS. Murine alveolar or peritoneal macrophages were obtained from bronchoalveolar lavage fluid or peritoneal lavage fluid 3 days after injection of $4 \%$ thioglycollate into the peritoneum, respectively. The cells were plated into 96-well plates at a density of $1 \times 10^{5}$ cells/well for all experiments except for fluorescent microscopy. After addition of L. pneumophila or MG132, the plate was centrifuged at $1000 \times g$ for $5 \mathrm{~min}$. For inhibition studies, the cells were pre-treated with CA074Me for $30 \mathrm{~min}$ before stimulation.

\section{Cytotoxicity assay}

Cell death was evaluated using a cytotoxicity detection kit (Roche Diagnostics GmbH, Mannheim, Germany) based on the measurement of LDH according to the manufacturer's instructions. To determine cytotoxicity, the value of samples from $1 \%$ Triton-X-treated cells was defined as $100 \%$ cell death.

\section{Optical microscopy}

Cells were stained with $0.2 \%$ trypan blue and trypan blue-positive and -negative cells were counted. To observe morphological findings, the cells were centrifuged at 1,000 $\times g$ for $1 \mathrm{~min}$, fixed onto slides and were then stained with Diff-Quik (Sysmex, Hyogo, Japan). Cellular morphological features were categorized by two cytologists. One hundred cells were counted three times and the results are presented as an average value.

\section{Fluorescent microscopy}

Mice macrophages were plated into a CultureWell chambered coverglass (Life Technologies, Carlsbad, CA) at a density of $1 \times 10^{3}$ cells/well (alveolar macrophages) or $1 \times$ $10^{5}$ cells/well (peritoneal macrophages) and were cultured in fresh medium overnight. After treatment, the cells were incubated with PBS containing $7.5 \mu \mathrm{g} / \mathrm{mL}$ Hoechst 33342 and $2 \mu \mathrm{g} / \mathrm{mL}$ PI for $30 \mathrm{~min}$. The preparation was observed using a Leica DM400B fluorescent microscope (Leica Microsystems GmbH, Wetzlar, Germany).

\section{Cathepsin B activity}

Cells were washed and homogenized at $4^{\circ} \mathrm{C}$ in lysis buffer as described previously [16]. Cell lysates $(10 \mu \mathrm{g})$ 
were incubated with $20 \mu \mathrm{M} \mathrm{z}$-Arg-Arg-MCA (Peptide Institute. Inc., Osaka, Japan) at $37^{\circ} \mathrm{C}$ for $2 \mathrm{~h}$ and the release of amino-4-methylcoumarin (AMC) was monitored using a SPECTRA Flour microplate reader (Tecan Japan Co., Ltd., Kanagawa, Japan). All experiments were performed in duplicate.

\section{Flow cytometry}

To evaluate cell death changes, cells were simultaneously stained with FITC-conjugated Annexin-V and the non-vital dye PI (Bender Medsystems, Vienna, Austria) to enable discrimination of intact cells (Annexin- $\left.\mathrm{V}^{-}, \mathrm{PI}^{-}\right)$, early apoptotic cells (Annexin- $\mathrm{V}^{+}, \mathrm{PI}^{-}$) and late apoptotic or necrotic cells $\left(\right.$ Annexin $\left.-\mathrm{V}^{+}, \mathrm{PI}^{+}\right)$. Cells were harvested after treatment, and $10^{4}$ cells per sample were analyzed using a FACSCalibur flow cytometer and CellQuest software (BD Biosciences Immunocytometry Systems, San Jose, CA).

\section{Western blotting}

Cells were harvested after treatment, and were washed and homogenized at $4^{\circ} \mathrm{C}$ in RIPA buffer. Cell lysates $(20-50 \mu \mathrm{g})$ were resolved by electrophoresis on a $12.5 \%$ polyacrylamide gel, and were transferred to a polyvinylidine difluoride membrane. After blocking the membrane in $10 \%$ FBS and $0.1 \%$ Tween-20 in Tris-buffered saline for $1 \mathrm{~h}$ at room temperature, blots were hybridized with primary antibodies overnight at $4^{\circ} \mathrm{C}$. After hybridization with a secondary antibody, the immunocomplexes were visualized using an ECL Western Blotting Detection System (GE Healthcare, Chalfont St. Giles, UK).

\section{HMGB-1 concentration}

The concentration of extracellular HMGB-1 was measured using an HMGB-1 ELISA Kit II (Shino Test, Kanagawa, Japan) according to the manufacturer's instructions. Briefly, $10 \mu \mathrm{L}$ of samples were added to an anti-HMBG-1 antibody-coated plate. After incubation at $37^{\circ} \mathrm{C}$ for $24 \mathrm{~h}$, the plate was incubated with peroxidaseconjugated anti-HMGB-1 monoclonal antibody for $2 \mathrm{~h}$ at room temperature. Color was developed using a 3,3',5,5'-tetramethylbenzidine and peroxidase solution. Absorbance was read at $450 \mathrm{~nm}$.

\section{Statistical analysis}

Cytotoxicity, morphological classification and HMGB-1 concentrations were expressed as means \pm SD. One-way analysis of variance was used to determine statistically significant differences between groups. Tukey's test was used to confirm differences between individual groups.

\section{Results}

A High dose of $L$. pneumophila induces necrotic cell death The levels of L. pneumophila used were calculated based on the ratio of bacteria to cells, and are expressed as multiplicity of infection (MOI). First, we assayed the time course of lactate dehydrogenase $(\mathrm{LDH})$ release into the culture medium following incubation of THP-1 cells with various levels of L. pneumophila (Figure 1A). L. pneumophila increased the levels of $\mathrm{LDH}$, as well as the percentage of trypan blue-positive cells (data not shown), in a dose- and time-dependent manner but not an apoptosis inducer, MG132 [17] (Figure 1B). Following $6 \mathrm{~h}$ of incubation with an MOI 500 of L. pneumophila, the cells showed characteristic features that are consistent with necrosis such as the loss of plasma membrane integrity and irregular and poorly-stained nuclei in contrast to MG132-treated apoptotic cells (Figure $1 C$ ). The necrotic cells partially appeared even when cells were infected by a low dose of bacteria, and the necrotic cells increased in a dose-dependent manner with addition of L. pneumophila (Figure 1D). Thus, an MOI 500 of bacteria was used in subsequent experiments to analyze the character of L. pneumophilainduced necrotic cells. In flow cytometry analysis with Annexin-V and PI binding, L. pneumophila-treated cells were divided into two clusters over time of treatment (Figure 1E). The cells in one group rapidly transformed into cells that were double-positive for Annexin- $V$ and PI that represented $30.5 \%$ of the total population at $2 \mathrm{~h}$. The second group of cells began to appear as Annexin$\mathrm{V}$-positive and PI-negative cells at $2 \mathrm{~h}$ and some of these cells were observed as double-positive cells at $6 \mathrm{~h}$. High mobility group box 1 (HMGB-1), known as a necrotic marker as well as a proinflammatory molecule, was also significantly increased in the culture medium after L. pneumophila stimulation (Figure 1F). These suggest that a high dose of L. pneumophila induces necrotic cell death.

\section{L. pneumophila-induced necrotic cell death is dependent on cathepsin B}

To examine the involvement of cathepsin B in L. pneumophila-induced necrotic cell death, we assayed the cellular level of pro-cathepsin B. The level of pro-cathepsin $B$ gradually diminished over time following infection, suggesting its processing into an active enzyme (Figure 2A). Pre-treatment with a specific cathepsin B inhibitor, CA074Me, at a concentration of 50 or $100 \mu \mathrm{M}$ decreased L. pneumophila-induced LDH by about $50 \%$ (Figure 2B). Thus, we used CA074Me at a concentration of $100 \mu \mathrm{M}$ in subsequent inhibitory experiments. This concentration of CA074Me alone did not affect all of the parameters in this study and bacterial growth. Microscopic observation clearly indicated fewer necrotic cells after CA074Me pretreatment than non-treated cells (Figure 2C). Instead, the number of intact cells, which had maintained a plasma membrane and a normally stained and shaped nucleus, and apoptotic cells seemed 
A

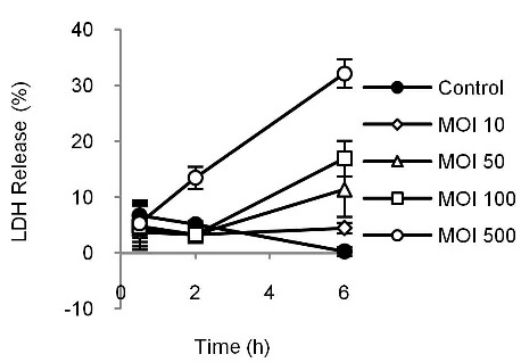

D

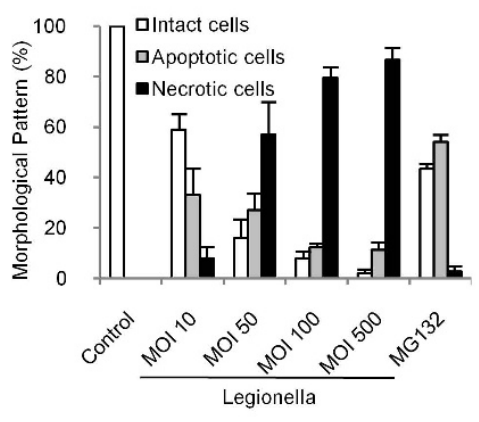

B

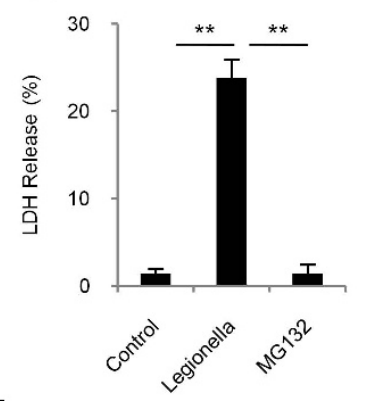

E

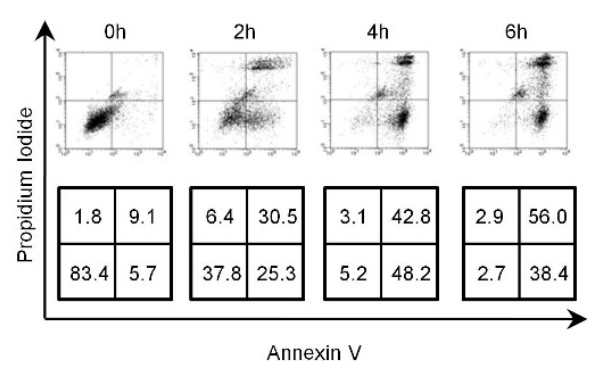

C
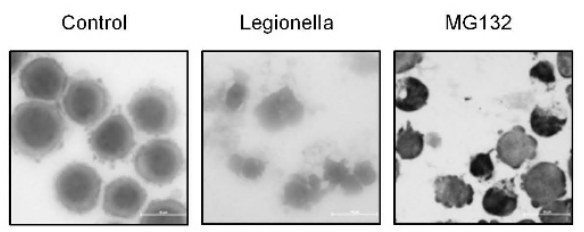

F

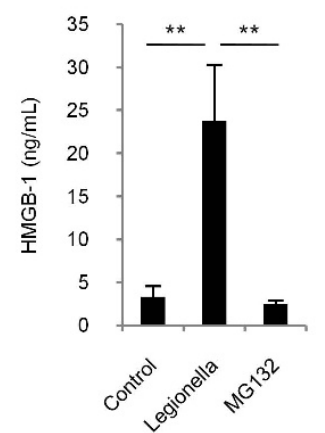

Figure 1 A high dose of L. pneumophila induces necrotic cell death in THP-1 cells. (A) Cytotoxicity of L. pneumophila was evaluated by assay of $L D H$ release from THP-1 cells. (B) LDH release after $6 \mathrm{~h}$-stimulation with an MOI 500 of $L$. pneumophila or $10 \mu \mathrm{M}$ of the apoptotic inducer MG132. (C) Diff-Quik staining after 6h-stimulation with an MOI 500 of L. pneumophila or MG132. L. pneumophila induced necrotic features such as loss of plasma membrane integrity and irregular and poorly-stained nuclei. The scale bar represents $20 \mu \mathrm{m}$. (D) Microscopic findings of the three cell groups identified following $6 \mathrm{~h}$ stimulation. L. pneumophila increased the percentage of necrotic cells in a dosedependent manner. In contrast, MG132 induced mainly apoptotic cells. (E) Flow cytometric analysis of THP-1 cells, infected with an MOI of 500 of L. pneumophila, and stained with Annexin-V and PI. The number at the bottom of each panel indicates the percentage of cells detected in that area. (F) Extracellular HMGB-1 after $6 \mathrm{~h}$ incubation following infection with an MOI of 500 of L. pneumophila, or with MG132. HMGB-1 was significantly increased by L. pneumophila but not by MG132. Values are expressed as means \pm SD. ${ }^{* *}$ indicates $p<0.01$.

to increase. The extracellular HMGB-1 was also significantly suppressed by CA074Me pretreatment compared to L. pneumophila stimulation alone (Figure 2D). These findings were also observed in the assay with another isolate (data not shown). These suggest that L. pneumophila-induced necrotic cell death is dependent on cathepsin B.

\section{Cathepsin B regulates both necrotic and apoptotic cell death}

Next, the effect of CA074Me on the type of cell was analyzed. CA074Me pretreatment decreased the percentage of necrotic cells to $50 \%$ or lower than that of non-treated cells (Figure 3A). Annexin-V- and PI-double-positive cells decreased and that the intact double-negative cells increased (Figure 3B). In MG132-treated cells, CA074Me pretreatment slightly suppressed the percentage of apoptotic cells (Figure 3C) and this effect was more clearly observed in flow cytometry (Figure 3D). These results indicated that cathepsin B is involved in the induction of both necrotic and apoptotic cell death.

\section{L. pneumophila-induced high activation of cathepsin $B$ is} involved in poly(ADP-ribose) polymerase (PARP) cleavage and cell death

To analyze the different functions of cathepsin B on cell death, we examined the time course of cathepsin B activation. Interestingly, L. pneumophila induced a rapid peak of cathepsin B activity that occurred within $2 \mathrm{~h}$ and subsequently dropped to approximately half of the peak value, whereas MG132 treatment induced a gradual elevation (Figure 4A). The high cathepsin B activation was also observed in other experiments within $6 \mathrm{~h}$. CA074Me almost completely inhibited the activation of cathepsin B (data not shown). Next, we examined the cleavage of PARP at $2 \mathrm{~h}$ with using CA074ME or a pancaspase inhibitor, zVADfmk (Figure 4B) because PARP cleavage can be observed in necrosis [18] and apoptosis 
A

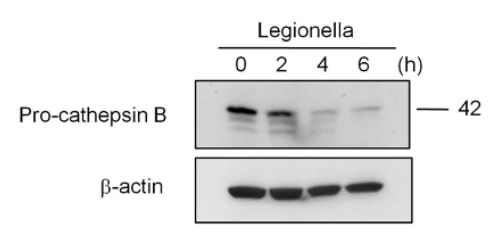

B

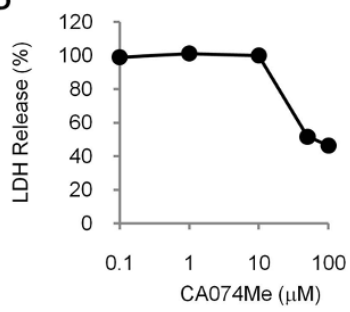

C

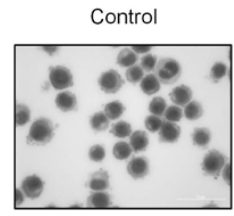

Legionella
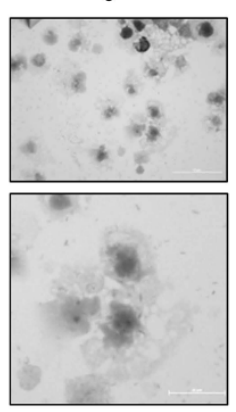

Legionella $+\mathrm{CA074Me}$

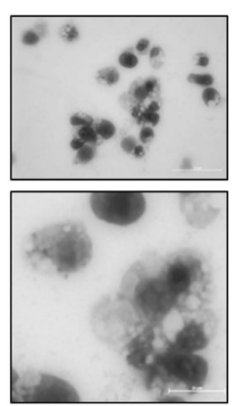

D

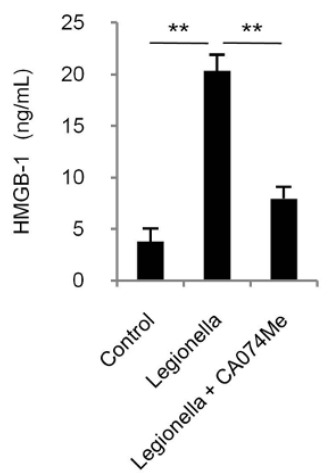

Figure 2 L. pneumophila-induced THP-1 cell death is cathepsin B-dependent. (A) Western blot analysis of pro-cathepsin B expression following stimulation with an MOI of 500 of L. pneumonia for the indicated times. (B) The effect of the specific cathepsin B inhibitor, CA074Me, on the L. pneumophila -induced LDH. CA074Me inhibited L. pneumophila-induced cytotoxicity at concentrations greater than $50 \mu \mathrm{M}$. (C) The microscopic findings after pretreatment with CA074Me $(100 \mu \mathrm{M})$ on L. pneumophila-induced necrotic cells. The top scale bar represents $50 \mu \mathrm{m}$ and the bottom represents $20 \mu \mathrm{m}$. (D) The effect of CA074 pretreatment on L. pneumophila-induced elevation of HMGB-1. Error bars in (B) and (D) represent means $\pm S D$. ** indicates $p<0.01$.

[19]. L. pneumophila dramatically enhanced the intensity of cleaved PARP compared to MG132 and this enhancement was attenuated more strongly by CA074Me than by zVADfmk. L. pneumophila-induced LDH was also significantly suppressed by pretreatment with CA074Me but not by pretreatment with zVADfmk (Figure 3C). These results suggest that L. pneumophila induces caspase-independent but cathepsin B-dependent PARP cleavage and cell death.

\section{Cathepsin B is involved in L. pneumophila-induced murine macrophage death}

We examined whether the cathepsin B-mediated, $L$. pneumophila-induced cell death also occurs in murine macrophages. A/J mouse alveolar or peritoneal macrophages were stained with $\mathrm{PI}$, which serves as a marker of plasma membrane permeability (Figure 5A). Most of the alveolar macrophages were PI-positive after stimulation with L. pneumophila. However, pretreatment with CA074Me markedly reduced the number of PI-positive cells. In the assay of peritoneal macrophages, the LDH level that was elevated by $L$. pneumophila was decreased by pretreatment with CA074Me (Figure 5B).

\section{Discussion}

In this paper, we studied cell death induced by L. pneumophila in macrophages and compared this death with that induced by an apoptotic agent. A high dose of L. pneumophila induced necrotic cell death in which cathepsin B played an important role in determination of the mechanism of cell death. This study reveals a novel aspect of the pathogenesis of Legionnaires' disease and identifies potential novel therapeutic targets for the regulation of inflammation.

Research regarding the mechanism of cell death related to L. pneumophila infection has focused mainly on apoptosis induced in monocytic cell lines or in murine macrophages with using a low dose of bacteria, such as an MOI 50 or lower $[7,9]$. On the other hand, Husmann and Johnson reported that infection with high multiplicity of L. pneumophila induce cytotoxicity in guinea pig peritoneal macrophages [20]. Similarly, the same dose in this study also induced necrotic cell death in mouse bonemarrow derived macrophages [11]. However, mechanisms of necrotic cell death were not determined.

Apoptosis is generally recognized as a non-inflammationproducing cell death [3], however, serious inflammation is 
A

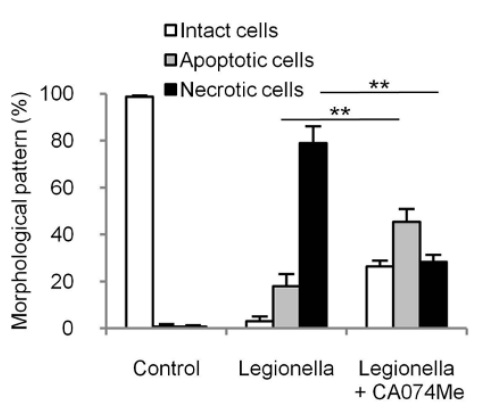

C

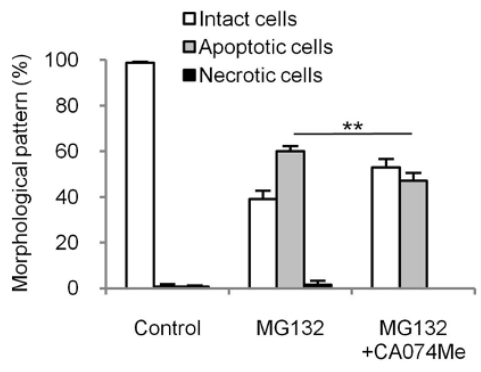

B

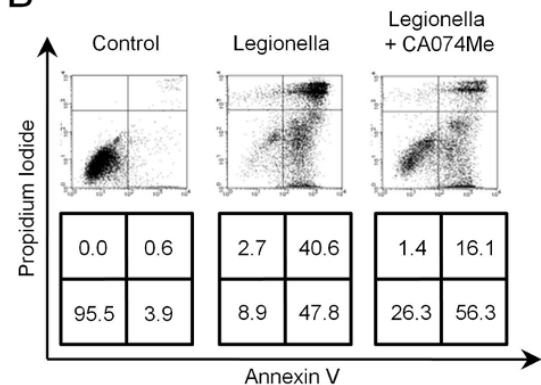

D

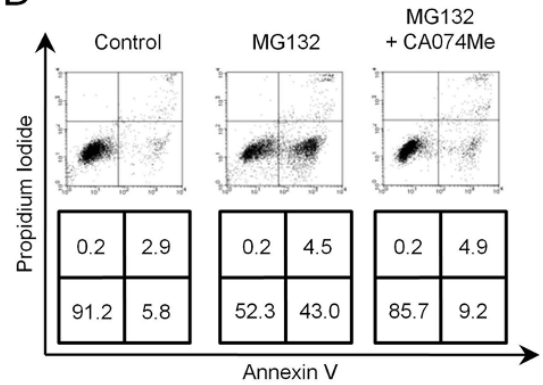

Figure 3 Cathepsin B regulates necrosis and apoptosis. (A) Classification of L. pneumophila-infected cells $6 \mathrm{~h}$ after stimulation based on morphological features. The percentage of necrotic cells was significantly decreased by pretreatment with CA074. (B) Annexin V and PI stained cells were evaluated by flow cytometry analysis $6 \mathrm{~h}$ after stimulation with L. pneumophila. The percentage of stained cells is indicated at the bottom of each panel. (C) Morphological classification of MG132-treated cells. MG132 induced-apoptotic cells were inhibited by pretreatment with CA074Me. (D) Flow cytometric analysis of MG132-treated cells stained with Annexin V and PI at 6 h. Values are expressed as means \pm SD. ** indicates $p<0.01$.

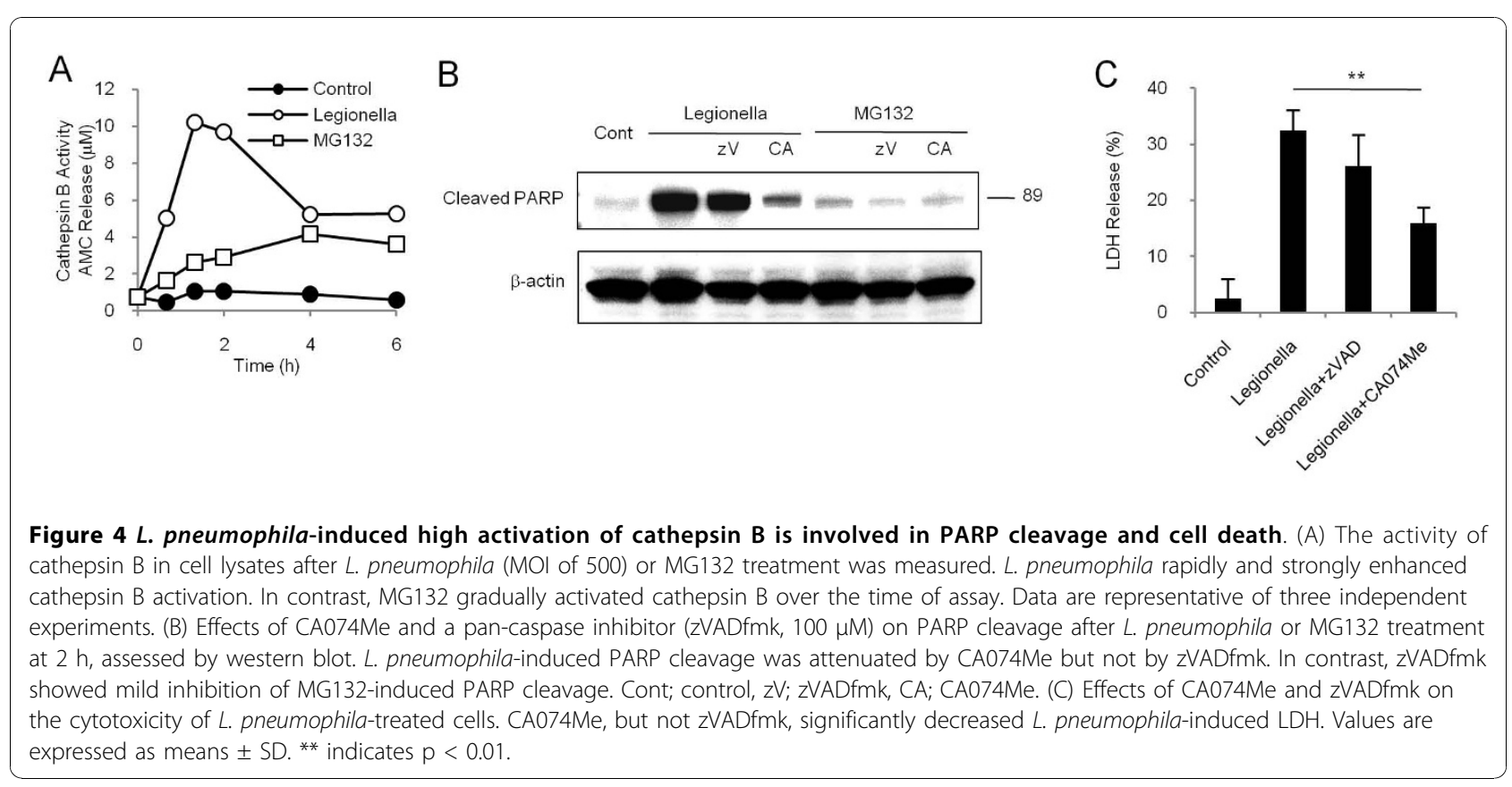


A
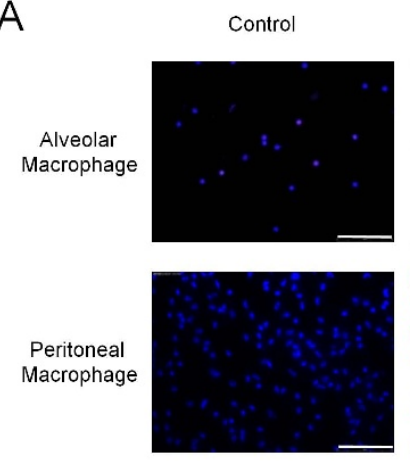

Legionella
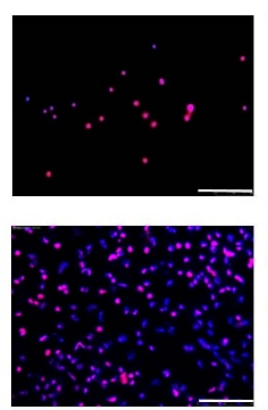
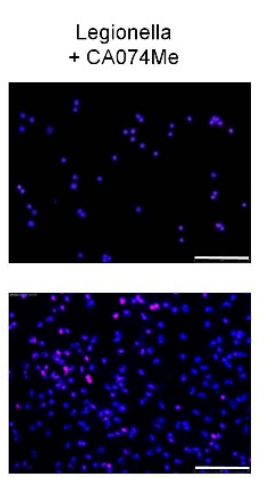

B

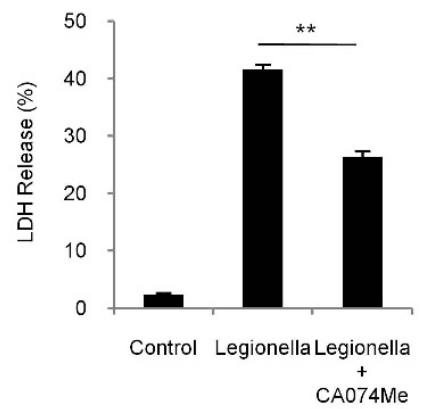

Figure 5 Cathepsin B is involved in L. pneumophila-induced murine macrophage death (A) Macrophages derived from the bronchoalveolar or peritoneal lavage fluid of A/J mice were stimulated with an $\mathrm{MOI} 500$ of L. pneumophila for $6 \mathrm{~h}$ followed by staining with Hoechst 33342 and PI. The PI-positive cells (red) represent necrotic cells. L. pneumophila infection increased, and pretreatment with CA074Me decreased, the number of PI-positive cells. The scale bar represents $75 \mu \mathrm{m}$. (B) LDH release $6 \mathrm{~h}$ after L. pneumophila-infection in peritoneal macrophages. CA074Me pretreatment had an inhibitory effect on L. pneumophila-induced cytotoxicity. Values are expressed as means \pm SD. ** indicates $\mathrm{p}<0.01$.

usually observed in patients with Legionnaires' disease. Since necrosis is known to induce inflammation, necrosis could potentially be involved in the pathogenesis of Legionnaires' disease. Necrotic cells appeared even after the treatment with a low dose of bacteria as shown in Figure 1D, and necrotic cells increased HMGB-1. Thus, the mixed type of cell death can be involved in the pathogenesis of Legionnaires' disease.

Apoptosis is a major programmed cell death and its signaling pathways and inducers have been well studied $[21,22]$. There is some evidence that necrosis, like apoptosis, is regulated by several enzymes [23] such as lysosomal enzymes, cathepsins [24]. For instance, cathepsin $\mathrm{B}$ mediates apoptosis induced by TRAIL in oral cancer cells [25] and necrosis induced by nigericin in THP-1 cells [26]. In this study, cathepsin B also regulated both apoptotic and necrotic cell death.

Thus, cathepsin B is a non-caspase protease that can lead to apoptosis and necrosis. However, the mechanistic details of cathepsin $B$ involvement in each type of cell death are still uncertain. The type of cell death mediated by lysosomal enzymes appears to depend on the magnitude of lysosomal permeabilization and the consequent release of lysosomal enzymes into the cytosol. Thus, the massive release of enzymes after complete lysosomal permeabilization induces necrotic cell death, whereas partial permeabilization with the release of lower levels of enzymes leads to apoptotic cell death [14]. L. pneumophila-induced high cathepsin B activity would support the possibility that complete lysosomal permeabilization had occurred, resulting in necrotic cell death. However, as pretreatment with CA074Me showed incomplete inhibition of cell death, reactive oxygen species [27] and multiple proteases such as calpains [28] and other cathepsins $[29,30]$ may be involved in L. pneumophila-induced necrotic cell death by crosstalking.

Recently, several cell death pathways have been newly identified. Pyroptosis is a caspase-1-dependent cell death and results in releasing inflammatory cytokines such as IL-1 $\beta$ and IL-18 [31]. It is reported that L. pneumophila induces pyroptosis [32,33]. Pyroptosis features plasma membrane permeability and nuclear condensation, however, cells in this study showed poor-stained nuclei which is mismatched to pyroptosis. In addition, in prescreening of inhibitory effect of cell death in this study, a specific caspase-1 inhibitor, zYVADcmk, had no effect on the LDH release (data not shown). Necroptosis or programmed necrosis, another type of cell death, is a necrotic cell death which is executed by enzymatic regulation such as receptor-interacting protein kinase 1, calpains and cathepsins [23]. The cell death in this study showed necrotic feature and cytotoxicity induced by L. pneumophila was independent of caspases including caspase- 1 but dependent of cathepsin B, suggesting that the cell death in this study was consistent with necroptosis rather than pyroptosis. However, our results with the immortalized cell line may need to be interpreted with caution in terms of cell death phenotypes as the immortalization process can affect cell death pathways.

In the cell death in the host response to L. pneumophila infection, flagellin is an important bacterial component as an inducer of pyroptosis [32]. Pyroptosis is also observed during Salmonella [34] or Shigella [35] 
infection, however, roles of bacterial flagellin on cell death pathways are different in these bacteria. Since we did not rule out the possibility of the involvement of flagellin and other components, we need further examination to reveal the relationship between bacterial components and cell death.

Cleaved PARP has been widely employed as a useful hallmark of apoptosis because PARP is processed by caspases [36]. However, L. pneumophila induced necrotic cell death in this study was accompanied by cleavage of PARP. This conflicting result can be attributed to excessive cathepsin $B$ activation because PARP can be processed not only by caspases but also by cathepsin $B$ and other proteases such as calpain, granzyme B and cathepsin G [18,37]. Indeed, L. pneumophila-induced cleaved PARP was attenuated by a cathepsin B inhibitor but not by a pan-caspase inhibitor, and the necrotic features of the cells were probably caused by indiscriminate degradation of cellular components by cathepsin $B$.

For the treatment of serious infectious diseases, it is important to regulate excessive inflammation. HMGB-1 is a cytokine that mediates strong systemic inflammatory responses, activates prototypical inflammatory responses in immune cells [38], and clinically causes acute lung injury [39]. Sepsis is a major example of severe inflammatory disease in which a vicious cycle involving HMGB-1 is implicated and elevated serum levels of HMGB-1 correlate with a worse prognosis in patients with sepsis [40]. Because severe cases of Legionnaires' disease can occur against which antibiotic therapy alone is insufficient even using antibiotics with high antibacterial activities [41], this excessive inflammation involving HMGB-1 induced by L. pneumophila is associated with the severity of the pathogenesis. Although further examination is necessary to determine whether inhibition of necrosis is actually beneficial for the treatment of Legionnaires' disease, the programmed necrosis induced by L. pneumophila that is described in this study may provide new information for the identification of therapeutic targets.

In conclusion, we report that a high dose of L. pneumophila induces cathepsin B-dependent necrosis in macrophages and that HMGB-1 release was also regulated by inhibition of necrotic cell death. The pattern of cathepsin $B$ activation was important for subsequent PARP cleavage and the type of cell death. Necrotic cell death can contribute to the pathogenesis of Legionnaires' disease and its severity and regulation of this cell death may provide some benefit in treatment of this disease.

\section{Abbreviations}

(HMGB-1): high-mobility group box 1; (PARP): poly(ADP-ribose) polymerase; (LDH): lactate dehydrogenase.

\section{Author details}

'Department of Laboratory Medicine, Nagasaki University Graduate School of Biomedical Sciences, 1-7-1 Sakamoto, Nagasaki, 851-2128, Japan. ${ }^{2}$ Second Department of Internal Medicine, Nagasaki University Graduate School of Biomedical Sciences, 1-7-1 Sakamoto, Nagasaki, 851-2128, Japan. ${ }^{3}$ Global COE Program, Nagasaki University, 1-7-1 Sakamoto, Nagasaki, 851-2128, Japan.

\section{Authors' contributions}

YM designed the study, carried out the molecular studies, the cell culture, the animal study, image analysis and data analysis, participated in the flow cytometry and drafted the manuscript. HH carried out the flow cytometry. $\mathrm{KY}, \mathrm{HH}, \mathrm{YYamad}$ and SKa participated in the design of the study and revised the manuscript. SN, MS, KI, HK, YYamam and SKo coordinated the study. All authors read and approved the final manuscript.

\section{Competing interests}

The authors declare that they have no competing interests.

Received: 15 August 2010 Accepted: 22 November 2010 Published: 22 November 2010

\section{References}

1. Friedman $\mathrm{H}$, Yamamoto $\mathrm{Y}$, Klein TW: Legionella pneumophila pathogenesis and immunity. Semin Pediatr Infect Dis 2002, 13(4):273-279.

2. Kumar S: Caspase function in programmed cell death. Cell Death Differ 2007, 14(1):32-43.

3. Zong WX, Thompson CB: Necrotic death as a cell fate. Genes Dev 2006, 20(1):1-15.

4. Phalipon A, Sansonetti PJ: Shigellosis: innate mechanisms of inflammatory destruction of the intestinal epithelium, adaptive immune response, and vaccine development. Crit Rev Immunol 2003, 23(5-6):371-401.

5. Guiney DG: The role of host cell death in Salmonella infections. Curr Top Microbiol Immunol 2005, 289:131-150.

6. Grover A, Taylor J, Troudt J, Keyser A, Sommersted K, Schenkel A, Izzo AA: Mycobacterial infection induces the secretion of high-mobility group box 1 protein. Cell Microbiol 2008, 10(6):1390-1404.

7. Gao LY, Abu Kwaik Y: Apoptosis in macrophages and alveolar epithelial cells during early stages of infection by Legionella pneumophila and its role in cytopathogenicity. Infect Immun 1999, 67(2):862-870.

8. Neumeister B, Faigle M, Lauber K, Northoff H, Wesselborg S: Legionella pneumophila induces apoptosis via the mitochondrial death pathway. Microbiology 2002, 148(Pt 11):3639-3650.

9. Santic M, Asare R, Doric M, Abu Kwaik Y: Host-dependent trigger of caspases and apoptosis by Legionella pneumophila. Infect Immun 2007, 75(6):2903-2913.

10. Kirby JE, Isberg RR: Legionnaires' disease: the pore macrophage and the legion of terror within. Trends Microbiol 1998, 6(7):256-258.

11. Kirby JE, Vogel JP, Andrews HL, Isberg RR: Evidence for pore-forming ability by Legionella pneumophila. Mol Microbiol 1998, 27(2):323-336.

12. Golstein P, Kroemer G: Cell death by necrosis: towards a molecular definition. Trends Biochem Sci 2007, 32(1):37-43.

13. Boya $\mathrm{P}$, Kroemer $\mathrm{G}$ : Lysosomal membrane permeabilization in cell death. Oncogene 2008, 27(50):6434-6451.

14. Guicciardi ME, Leist M, Gores GJ: Lysosomes in cell death. Oncogene 2004, 23(16):2881-2890.

15. Saijo T, Izumikawa K, Takazono T, Kosai K, Kurihara S, Nakamura S, Imamura Y, Miyazaki T, Seki M, Kakeya H, et al: A case of Legionella pneumophila pneumonia followed by invasive aspergillosis. Jpn J Infect Dis 2008, 61(5):379-381.

16. Yamada Y, Sugahara K, Tsuruda K, Nohda K, Mori N, Hata T, Maeda T, Hayashibara T, Joh T, Honda M, et al: Lactacystin activates FLICE (caspase 8) protease and induces apoptosis in Fas-resistant adult T-cell leukemia cell lines. Eur J Haematol 2000, 64(5):315-322.

17. He Q, Huang Y, Sheikh MS: Proteasome inhibitor MG132 upregulates death receptor 5 and cooperates with Apo2L/TRAIL to induce apoptosis in Bax-proficient and -deficient cells. Oncogene 2004, 23(14):2554-2558.

18. Gobeil S, Boucher CC, Nadeau D, Poirier GG: Characterization of the necrotic cleavage of poly(ADP-ribose) polymerase (PARP-1): implication of lysosomal proteases. Cell Death Differ 2001, 8(6):588-594. 
19. Lazebnik YA, Kaufmann SH, Desnoyers S, Poirier GG, Earnshaw WC: Cleavage of poly(ADP-ribose) polymerase by a proteinase with properties like ICE. Nature 1994, 371(6495):346-347.

20. Husmann LK, Johnson W: Cytotoxicity of extracellular Legionella pneumophila. Infect Immun 1994, 62(5):2111-2114.

21. Hasegawa H, Yamada Y, Komiyama K, Hayashi M, Ishibashi M, Sunazuka T, Izuhara T, Sugahara K, Tsuruda K, Masuda M, et al: A novel natural compound, a cycloanthranilylproline derivative (Fuligocandin B), sensitizes leukemia cells to apoptosis induced by tumor necrosis factor related apoptosis-inducing ligand (TRAIL) through 15-deoxy-Delta 12, 14 prostaglandin J2 production. Blood 2007, 110(5):1664-1674.

22. Hasegawa H, Yamada Y, Komiyama K, Hayashi M, Ishibashi M, Yoshida T, Sakai T, Koyano T, Kam TS, Murata K, et al: Dihydroflavonol BB-1, an extract of natural plant Blumea balsamifera, abrogates TRAIL resistance in leukemia cells. Blood 2006, 107(2):679-688.

23. Galluzzi L, Kroemer G: Necroptosis: a specialized pathway of programmed necrosis. Cell 2008, 135(7):1161-1163.

24. Salvesen GS: A lysosomal protease enters the death scene. J Clin Invest 2001, 107(1):21-22.

25. Nagaraj NS, Vigneswaran N, Zacharias W: Cathepsin B mediates TRAILinduced apoptosis in oral cancer cells. J Cancer Res Clin Oncol 2006, 132(3):171-183.

26. Hentze $H$, Lin XY, Choi MS, Porter AG: Critical role for cathepsin B in mediating caspase-1-dependent interleukin-18 maturation and caspase1 -independent necrosis triggered by the microbial toxin nigericin. Cell Death Differ 2003, 10(9):956-968.

27. Morgan MJ, Kim YS, Liu ZG: TNFalpha and reactive oxygen species in necrotic cell death. Cell Res 2008, 18(3):343-349.

28. Pignol B, Auvin S, Carre D, Marin JG, Chabrier PE: Calpain inhibitors and antioxidants act synergistically to prevent cell necrosis: effects of the novel dual inhibitors (cysteine protease inhibitor and antioxidant) BN 82204 and its pro-drug BN 82270. J Neurochem 2006, 98(4):1217-1228.

29. Artal-Sanz M, Samara C, Syntichaki P, Tavernarakis N: Lysosomal biogenesis and function is critical for necrotic cell death in Caenorhabditis elegans. $J$ Cell Biol 2006, 173(2):231-239.

30. Yamashima T: Ca2+-dependent proteases in ischemic neuronal death: a conserved 'calpain-cathepsin cascade' from nematodes to primates. Cell Calcium 2004, 36(3-4):285-293.

31. Labbe K, Saleh M: Cell death in the host response to infection. Cell Death Differ 2008, 15(9):1339-1349.

32. Molofsky AB, Byrne BG, Whitfield NN, Madigan CA, Fuse ET, Tateda K, Swanson MS: Cytosolic recognition of flagellin by mouse macrophages restricts Legionella pneumophila infection. J Exp Med 2006, 203(4):1093-1104.

33. Silveira TN, Zamboni DS: Pore formation triggered by Legionella spp. is an Nlrc4 inflammasome-dependent host cell response that precedes pyroptosis. Infect Immun 2010, 78(3):1403-1413.

34. Fink SL, Cookson BT: Pyroptosis and host cell death responses during Salmonella infection. Cell Microbiol 2007, 9(11):2562-2570.

35. Suzuki T, Franchi L, Toma C, Ashida H, Ogawa M, Yoshikawa Y, Mimuro H, Inohara N, Sasakawa C, Nunez G: Differential regulation of caspase-1 activation, pyroptosis, and autophagy via Ipaf and ASC in Shigellainfected macrophages. PLoS Pathog 2007, 3(8):e111.

36. Schreiber V, Dantzer F, Ame JC, de Murcia G: Poly(ADP-ribose): novel functions for an old molecule. Nat Rev Mol Cell Biol 2006, 7(7):517-528.

37. Chaitanya GV, Babu PP: Differential PARP cleavage: an indication of heterogeneous forms of cell death and involvement of multiple proteases in the infarct of focal cerebral ischemia in rat. Cell Mol Neurobiol 2009, 29(4):563-573.

38. Lotze MT, Tracey KJ: High-mobility group box 1 protein (HMGB1): nuclear weapon in the immune arsenal. Nat Rev Immunol 2005, 5(4):331-342.
39. Ueno H, Matsuda T, Hashimoto S, Amaya F, Kitamura Y, Tanaka M, Kobayashi A, Maruyama I, Yamada S, Hasegawa N, et al: Contributions of high mobility group box protein in experimental and clinical acute lung injury. Am J Respir Crit Care Med 2004, 170(12):1310-1316.

40. Dumitriu IE, Baruah P, Manfredi AA, Bianchi ME, Rovere-Querini P: HMGB1: guiding immunity from within. Trends Immunol 2005, 26(7):381-387.

41. Higa F, Akamine M, Haranaga S, Tohyama M, Shinzato T, Tateyama M, Koide M, Saito A, Fujita J: In vitro activity of pazufloxacin, tosufloxacin and other quinolones against Legionella species. J Antimicrob Chemother 2005, 56(6):1053-1057

doi:10.1186/1465-9921-11-158

Cite this article as: Morinaga et al:: Legionella pneumophila induces cathepsin B-dependent necrotic cell death with releasing high mobility group box1 in macrophages. Respiratory Research 2010 11:158.

\section{Submit your next manuscript to BioMed Central and take full advantage of:}

- Convenient online submission

- Thorough peer review

- No space constraints or color figure charges

- Immediate publication on acceptance

- Inclusion in PubMed, CAS, Scopus and Google Scholar

- Research which is freely available for redistribution

Submit your manuscript at www.biomedcentral.com/submit
Biomed Central 